\title{
Outcomes of contemporary imaging-guided management of sinus of Valsalva aneurysms
}

\author{
Bo Xu ${ }^{1}$, Duygu Kocyigit ${ }^{1}$, Carlos Godoy-Rivas ${ }^{2}$, Jorge Betancor ${ }^{3}$, L. Leonardo Rodriguez ${ }^{1}$, Venu Menon ${ }^{4}$, \\ Wael Jaber ${ }^{1}$, Richard Grimm ${ }^{1}$, Scott D. Flamm ${ }^{5}$, Paul Schoenhagen ${ }^{5}$, Lars G. Svensson ${ }^{6}$, Brian P. Griffin ${ }^{1}$ \\ ${ }^{1}$ Section of Cardiovascular Imaging, Robert and Suzanne Tomsich Department of Cardiovascular Medicine, Sydell and Arnold Miller Family Heart, \\ Vascular and Thoracic Institute, Cleveland Clinic, Cleveland, OH, USA; ${ }^{2}$ University of Connecticut Health Center, Farmington, CT, USA; ${ }^{3}$ Aventura \\ Hospital and Medical Center, Aventura, FL, USA; ${ }^{4}$ Section of Clinical Cardiology, Robert and Suzanne Tomsich Department of Cardiovascular \\ Medicine, Sydell and Arnold Miller Family Heart, Vascular and Thoracic Institute, Cleveland Clinic, Cleveland, OH, USA; ${ }^{5} \mathrm{Cardiovascular}$ Imaging \\ Laboratory, Imaging Institute, and Heart, Vascular and Thoracic Institute, Cleveland Clinic, Cleveland, OH, USA; ${ }^{6}$ Department of Thoracic and \\ Cardiovascular Surgery, Sydell and Arnold Miller Family Heart, Vascular and Thoracic Institute, Cleveland Clinic, Cleveland, OH, USA \\ Contributions: (I) Conception and design: B Xu, J Betancor; (II) Administrative support: None; (III) Provision of study material or patients: None; (IV) \\ Collection and assembly of data: B Xu, J Betancor, D Kocyigit, C Godoy-Rivas; (V) Data analysis and interpretation: All authors; (VI) Manuscript \\ writing: All authors; (VII) Final approval of manuscript: All authors. \\ Correspondence to: Bo Xu, MB, BS (Hons), FRACP, FACC, FASE. Section of Cardiovascular Imaging, Robert and Suzanne Tomsich Department of \\ Cardiovascular Medicine, Sydell and Arnold Miller Family Heart, Vascular and Thoracic Institute, Cleveland Clinic, 9500 Euclid Avenue, Desk J1-5, \\ Cleveland, OH 44195, USA. Email: xub@ccf.org.
}

Background: Sinus of Valsalva aneurysms (SVAs) are rare. We assessed the role of multimodality imaging in guiding the contemporary management.

Methods: A single-center retrospective cohort study over a 20-year period was performed.

Results: Between January 1997 and June 2017, 103 patients were diagnosed with SVAs (median age: 58 years). Eighty patients presented with non-ruptured SVAs, and 23 with ruptured SVAs. Seventy-six patients underwent surgery, and 27 were conservatively managed. The median durations of follow-up were: 48 months (surgical group) vs. 37.5 months (conservative group). There was no mortality directly attributable to SVA surgery. There were no late complications in the conservative group. Transthoracic echocardiography (TTE) was the first-line imaging investigation (100.0\% in surgical group vs. $92.6 \%$ in conservative group, $\mathrm{P}=0.019$ ). Additional imaging studies included: (I) transesophageal echocardiography (TEE): $93.4 \%$ in surgical group vs. $22.2 \%$ in conservative group, $\mathrm{P}<0.001$; (II) multi-detector cardiac computed tomography (MDCT): $61.8 \%$ in surgical group vs. $37.0 \%$ in conservative group, $\mathrm{P}=0.041$; (III) cardiac magnetic resonance (CMR): $22.4 \%$ in surgical group vs. $14.8 \%$ in conservative group, $\mathrm{P}=0.579$. At diagnosis, SVA diameters were: TTE: $4.80 \mathrm{~cm}$ (range, $3.30 \mathrm{~cm}$ ); TEE: $5.40 \mathrm{~cm}$ (range, $4.00 \mathrm{~cm}$ ); MDCT: 5.20 cm (range, $3.90 \mathrm{~cm}$ ); CMR: $4.80 \mathrm{~cm}$ (range, $3.70 \mathrm{~cm}$ ).

Conclusions: In a 20-year cohort, proper selection for surgery and conservative management resulted in excellent outcomes for SVAs. TTE was the first-line imaging investigation for assessment of SVAs, although many patients underwent an additional imaging investigation. The contemporary outcomes of imagingguided SVA management were excellent.

Keywords: Sinus of Valsalva aneurysm (SVA); transthoracic echocardiography; multidetector cardiac computed tomography; cardiac magnetic resonance imaging; multimodality imaging; cardiac surgery

Submitted Jul 10, 2020. Accepted for publication Sep 08, 2020.

doi: $10.21037 / \mathrm{cdt}-20-630$

View this article at: http://dx.doi.org/10.21037/cdt-20-630 


\section{Introduction}

Sinus of Valsalva aneurysms (SVAs) refer to dilatation of one of the aortic sinuses, between the aortic annulus and sinotubular junction (1). Since they often present as incidental findings on routine cardiothoracic imaging, the assessment of their true incidence is difficult. The limited epidemiological data are mostly derived from single-center studies exploring prevalence of ruptured SVAs, which have been reported to range between $0.23-0.96 \%$ among subjects undergoing cardiovascular surgeries $(2,3)$. Contemporary data on the diagnosis and management outcomes of SVAs are limited. The role of echocardiography and multimodality cardiac imaging in the diagnosis and management of SVAs is not well defined in the literature. We investigated the contemporary management of this rare condition over a 20 -year period and present the following article in accordance with the STROBE reporting checklist (available at http://dx.doi.org/10.21037/ cdt-20-630).

\section{Methods}

\section{Study population}

A retrospective cohort study was performed using the electronic medical records and echocardiographic database at our center. Consecutive patients with a diagnosis of SVA, between January 1997 and June 2017 were included. SVA is a unique entity with asymmetric dilatation of usually one of the aortic sinuses. The diagnosis was made by an experienced imaging cardiologist, incorporating imaging data from available echocardiographic and tomographic studies. For surgically managed patients, the diagnosis was confirmed at time of surgery.

Transthoracic echocardiography (TTE) was the firstline imaging modality. Transesophageal echocardiography (TEE), multidetector computed tomography (MDCT) and cardiac magnetic resonance (CMR) imaging were utilized as complementary imaging modalities to TTE. On TTE, SVA diameter was measured perpendicular to the long-axis of the ascending aorta in the parasternal long-axis view at enddiastole using the leading edge-to-leading edge convention (1). On TEE, SVA was imaged on mid-esophageal long-axis (at $\left.120-150^{\circ}\right)$ and short-axis $\left(30-60^{\circ}\right)$ views (1). MDCT examinations were dedicated, gated contrast-enhanced examinations. For CMR, three-dimensional navigatorgated whole heart sequence was used for assessment of thoracic aortic anatomy. On tomographic imaging with MDCT/CMR, SVA diameter was measured from sinus-to- sinus, using the double-oblique technique on multi-planar reconstruction (1) (Figure 1).

Patients diagnosed with SVA were divided into two groups based on the management strategy: surgical $v s$. conservative management. A case was included in the surgical management group if the patient underwent specific surgery for SVA, with findings recorded in surgical reports. Although dedicated guidelines for the management of SVAs are lacking, indications for surgical management of SVAs were inferred from aortic disease and valvular heart disease guidelines (1). These include: (I) aortic root size $\geq 5.5 \mathrm{~cm}$; (II) annual aortic root growth rate $>0.5$ $\mathrm{cm}$; (III) presence of an indication is present for aortic valve replacement due to severe aortic stenosis or aortic regurgitation when the aortic root size $\geq 4.5 \mathrm{~cm}$; (IV) aortic root size $\geq 5.0 \mathrm{~cm}$ in bicuspid aortopathy with additional risk factors; (V) aortic cross-sectional area/height ratio $>10$ $\mathrm{cm}^{2} / \mathrm{m}$ in bicuspid aortopathy or heritable thoracic aortic disease (1). The indications for conservative management of SVAs included: (I) SVA did not reach surgical size/stable on follow-up imaging (63\%); (II) advanced age and severe comorbidities (30.0\%); (III) patient refusal of surgery (7.0\%).

The study was approved by the institutional review board of the Cleveland Clinic (IRB number: 18-1445), and was compliant with the HIPAA (Health Insurance Portability and Accountability Act) regulations. The study was conducted in accordance with the Declaration of Helsinki (as revised in 2013).

\section{Data collection and follow-up}

Demographic variables and detailed clinical variables were collected through review of the electronic medical records. Imaging data were obtained from the echocardiographic, computed tomography and CMR imaging databases, which were reviewed by experienced cardiovascular and thoracic imaging physicians. The main outcomes were SVA-related mortality and all-cause mortality at follow-up. Surgically managed SVA patients were followed up additionally for early ( $\leq 30$ days from surgery) and late post-operative complications and surgery-related mortality. Follow-up was obtained by review of clinic follow-up data via electronic medical records. Data regarding deaths were obtained by a search of electronic medical records and online obituaries.

\section{Statistical analysis}

One sample Kolmogorov-Smirnov test was used to test whether the parameters had normal distribution. Mean 


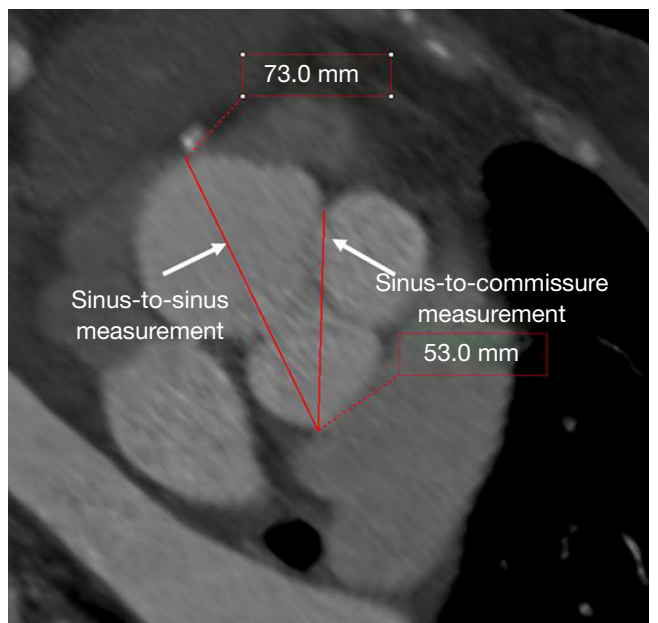

Figure 1 An illustrative example of a large right sinus of Valsalva aneurysm is shown to demonstrate sinus-to-sinus vs. sinus-tocommissure methods for measuring sinuses of Valsalva diameters by multidetector cardiac computed tomography.

\pm standard deviation presentation was used for normally distributed continuous parameters, which were compared using independent samples $t$-test. Median (range, defined as the difference between the maximum and the minimum values) was used for skewed continuous parameters, which were compared using Mann-Whitney U test. Categorical data were expressed in terms of absolute count and percentage of cohort. All percentages were presented relative to the total number of patients with available information, and were compared using Chi-squared test or Fisher's exact test. Binomial logistic regression was used to evaluate factors associated with SVA rupture. Longterm survival was evaluated with Kaplan-Meier survival analysis and compared using log-rank test. Cox regression analysis was performed to identify independent predictors of survival at follow-up in univariable and multivariable analyses. Results were reported as hazard ratio (HR) or odds ratio $(\mathrm{OR})$ and $95 \%$ confidence intervals (CIs). Statistical analyses were performed using SPSS statistical software (IBM Corp. Released 2015. IBM SPSS Statistics for Windows, Version 23.0. Armonk, NY: IBM Corp.) A twotailed $\mathrm{P}$ value $<0.05$ was considered statistically significant.

\section{Results}

Between January 1997 and June 2017, 103 patients were diagnosed with SVAs at our center (Figure 2). The median age was 58 years, with the majority being male $(68.9 \%)$

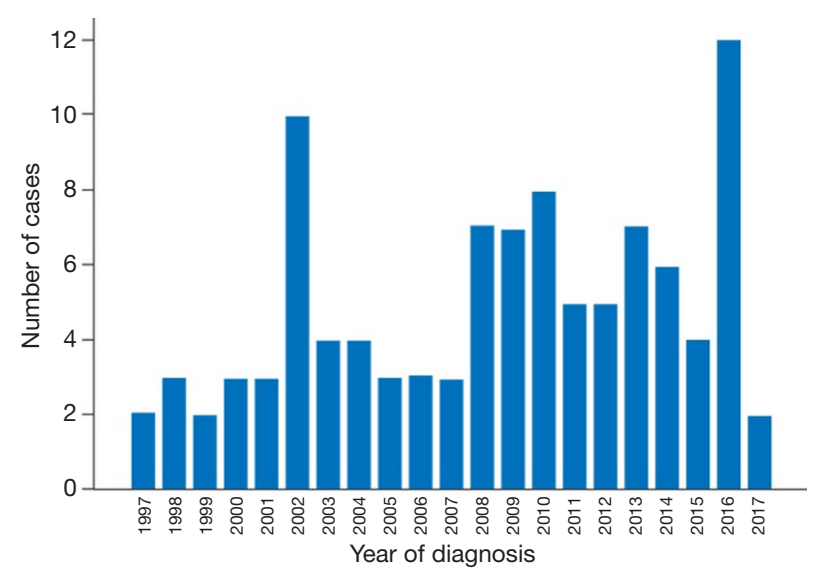

Figure 2 Distribution of the number of SVAs diagnosed per year over the study period between 1997 and 2017. SVA, sinus of Valsalva aneurysm.

and Caucasian (83.5\%) (Table 1). Conservatively managed patients $(\mathrm{n}=76)$ were significantly older than surgically managed patients $(\mathrm{n}=27)$ (median: 67 vs. 57 years, $\mathrm{P}=0.024)$. A significantly higher proportion of surgically managed patients had a murmur on physical examination $(67.1 \%$ vs. 44.4\%, $\mathrm{P}=0.036$ ) and complained of dyspnea (at rest and/ or with exertion) at presentation $(51.3 \%$ vs. $7.4 \%, \mathrm{P}<0.001)$ (Table 1). Congenital heart disease was more common in the surgical group, though it did not reach statistical significance $(55.3 \%$ vs. $37.0 \%$ in the conservative group, $\mathrm{P}=0.121)$ (Table 1).

Right coronary SVA was the commonest type (56.3\%), followed by non-coronary SVA $(35.9 \%)$ and left coronary SVA (7.8\%) (Figure 3). Five patients in the right coronary SVA group had another sinus involvement: accompanying non-coronary SVA in 2 patients, and left coronary SVA in 3 patients. At diagnosis, most SVAs were non-ruptured (77.7\%). Significantly more patients in the surgically managed group presented with ruptured SVA (27.6\% vs. $7.4 \%$ in the conservative group, $\mathrm{P}=0.033$ ) (Table 2).

\section{Surgically managed SVA patients}

Seventy-six patients were surgically managed (73.8\%), consisting of patch repair (54\%), aortic root replacement (52.6\%), plication $(13.2 \%)$, aortic valve repair $(11.8 \%)$, fistula ligation (4\%) and excision (2.6\%). Twenty-one patients $(27.6 \%)$ presented with ruptured SVAs.

The most common associated congenital heart disease included: bicuspid aortic valve (BAV) $(n=20,26.3 \%)$, 
Table 1 Demographic and clinical characteristics of the study cohort

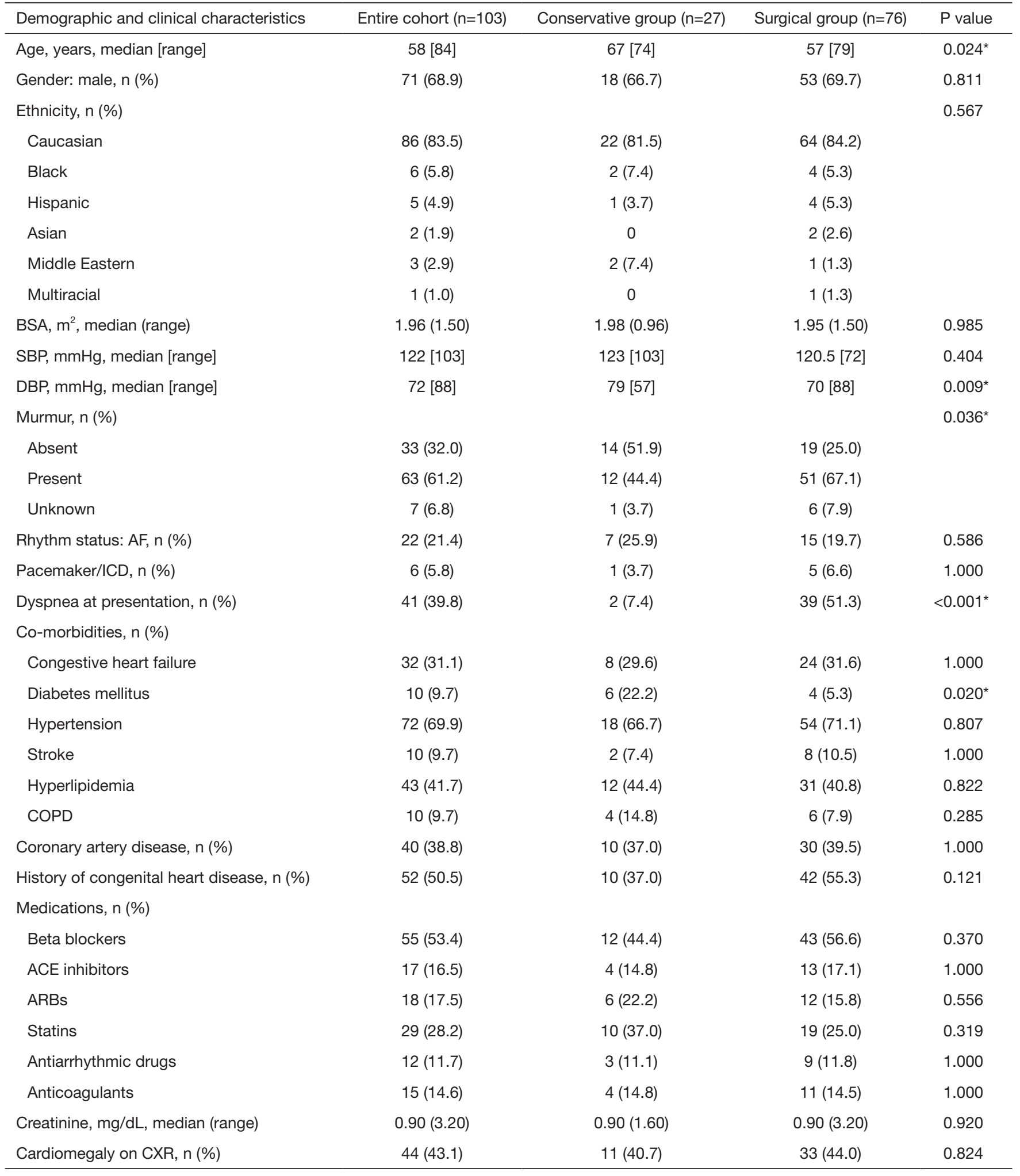

*, $P$ value $<0.05$ denotes statistical significance. ACE, angiotensin-converting enzyme; AF, atrial fibrillation; ARB, angiotensin-II receptor blocker; BSA, body surface area; COPD, chronic obstructive pulmonary disease; CXR, chest X-ray; ICD, implantable cardioverter defibrillator; SBP, systolic blood pressure; DBP, diastolic blood pressure. 


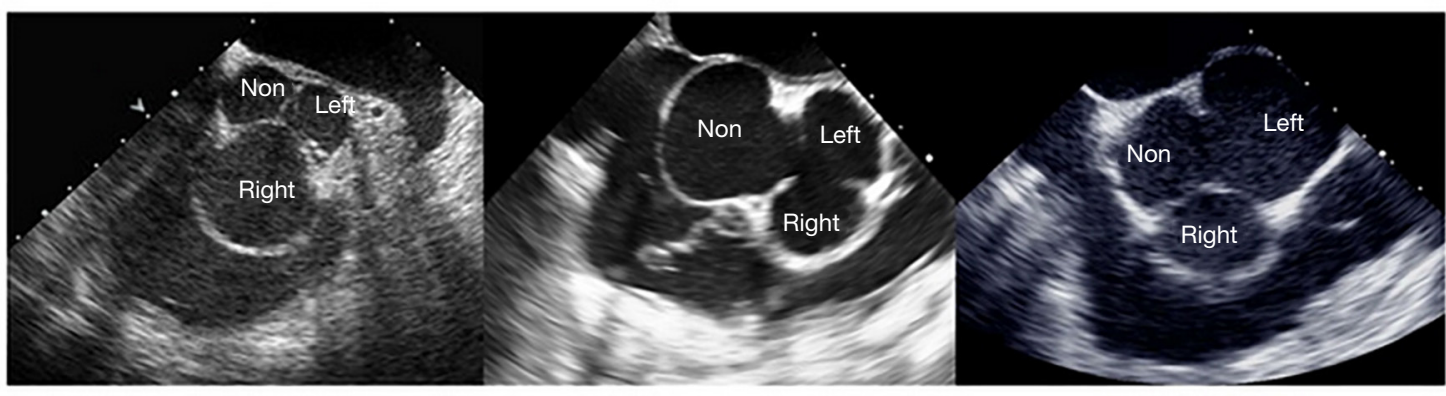

\begin{tabular}{lccc}
\hline & $\begin{array}{c}\text { Right coronary SVA } \\
(\mathrm{n}=58)\end{array}$ & $\begin{array}{c}\text { Non-coronary SVA } \\
(\mathrm{n}=37)\end{array}$ & $\begin{array}{c}\text { Left coronary SVA } \\
(\mathrm{n}=8)\end{array}$ \\
\hline Ruptured & $12(20.7 \%)$ & $11(29.7 \%)$ & 0 \\
\hline Non-ruptured & $46(79.3 \%)$ & $26(70.3 \%)$ & $8(100 \%)$ \\
Conservatively managed & $15(25.9 \%)$ & $11(29.7 \%)$ & $1(12.5 \%)$ \\
Surgically managed & $43(74.1 \%)$ & $26(70.3 \%)$ & $7(87.5 \%)$ \\
\hline
\end{tabular}

Figure 3 Distribution of the different types of SVAs in the study cohort. The cases are further stratified by presence of rupture at presentation and management strategy (conservative $v s$. surgical). Five patients in the right coronary SVA group had another sinus involvement: accompanying non-coronary SVA in 2 patients, and left coronary SVA in 3 patients. SVA, sinus of Valsalva aneurysm.

ventricular septal defect (VSD) $(\mathrm{n}=16,21.1 \%)$, patent foramen ovale $(\mathrm{n}=13,17.1 \%)$ and atrial septal defect $(\mathrm{n}=4,5.3 \%)$. These existed as either a single anomaly accompanying the SVA or in combination with other forms of congenital heart disease. The most common combinations of congenital heart disease associated with SVAs were: VSD and patent foramen ovale $(\mathrm{n}=3,3.9 \%)$ and BAV and patent foramen ovale ( $\mathrm{n}=3,3.9 \%$ ) (Table S1).

Early and late post-operative complications are summarized in Table S2. The only in-hospital death was caused by a case of endocarditis related to left ventricular assist device, which was complicated by septic cerebral emboli (1.3\%). There was no mortality directly related to SVA surgery. The median follow-up duration in the surgical group was 48 months (range, 396 months). At follow-up, six patients died in the surgical group from non-SVA related causes: tonsil cancer in one patient, pneumonia in one patient, breast cancer in one patient, post-operative sepsis after nephrectomy and adrenalectomy in one patient, and two patients died from an unknown cause. The KaplanMeier survival curve is shown in Figure 4, and the estimated survival rate at 31 months was $94.0 \%$ (Figure 4).

\section{Conservatively managed SVA patients}

Twenty-seven patients were conservatively managed $(26.2 \%)$. The median follow-up duration in the conservatively managed group was 37.5 months (range, 227 months). Two patients with a ruptured SVA were in the conservative group: one patient was a 49-year-old male with abdominal sepsis and mediastinal lymphadenopathy who died from pulseless electrical activity cardiac arrest, prior to consideration of intervention for SVA; the other patient was an apparently well 38-year-old female with an incidentally noted murmur, who was found to have a ruptured SVA involving the non-coronary sinus. This patient did not undergo surgery for SVA during the study period.

Although mortality at follow-up was higher in the conservatively managed group $(30.8 \%$ vs. $9.6 \%$ in the surgical group, $\mathrm{P}=0.021)$, none of the deaths were directly related to SVA. Eight patients (30.8\%) died from non-SVA related causes: four died from malignancies (colon cancer in two patients, lung cancer in one patient, and multiple myeloma in one patient), two died from gastrointestinal bleeding, one died due to pre-surgery cardiac arrest and one died from an unknown cause. The estimated survival rate was $81.4 \%$ at 26 months, and compared to surgically managed patients, overall survival was significantly lower in the conservatively managed group $(\mathrm{P}=0.021)$ (Figure 4).

\section{Imaging investigations in managing SVA}

TTE was the first-line imaging investigation: $100.0 \%$ (surgical group) and 92.6\% (conservative group). Additional 
Table 2 Multi-modality cardiovascular imaging assessment of SVAs in the study cohort

\begin{tabular}{|c|c|c|c|c|}
\hline Imaging characteristics & Entire cohort $(n=103)$ & Conservative group $(\mathrm{n}=27)$ & Surgical group $(n=76)$ & $P$ value \\
\hline TTE & $101(98.1)$ & $25(92.6)$ & $76(100.0)$ & \\
\hline MDCT & $1(1.0)$ & $1(3.7)$ & 0 & \\
\hline CMR & $1(1.0)$ & $1(3.7)$ & 0 & \\
\hline TTE & $102(99.0)$ & $26(96.3)$ & $76(100.0)$ & 0.262 \\
\hline TEE & $77(74.8)$ & $6(22.2)$ & $71(93.4)$ & $<0.001^{*}$ \\
\hline MDCT & 57 (55.3) & $10(37.0)$ & $47(61.8)$ & $0.041^{*}$ \\
\hline CMR & $21(20.4)$ & $4(14.8)$ & $17(22.4)$ & 0.579 \\
\hline Location of SVA, n (\%) & & & & 0.601 \\
\hline LCS & $8(7.8)$ & $1(3.7)$ & $7(9.2)$ & \\
\hline $\mathrm{RCS}^{\star \star}$ & $58(56.3)$ & $15(55.6)$ & $43(56.6)$ & \\
\hline NCS & 37 (35.9) & $11(40.7)$ & $26(34.2)$ & \\
\hline Rupture Status of SVA, n (\%) & & & & $0.033^{*}$ \\
\hline Non-ruptured & $80(77.7)$ & $25(92.6)$ & $55(72.4)$ & \\
\hline Ruptured & $23(22.3)$ & $2(7.4)$ & $21(27.6)$ & \\
\hline SVA diameter on TTE, cm, median (range) & $4.80(3.30)$ & $4.20(3.20)$ & $4.90(3.10)$ & $0.018^{*}$ \\
\hline
\end{tabular}

*, $\mathrm{P}$ value $<0.05$ denotes statistical significance; ${ }^{* *}, 5$ patients in the right coronary SVA group had another sinus involvement: accompanying non-coronary SVA in 2 patients, and left coronary SVA in 3 patients. CMR, cardiac magnetic resonance imaging; LCS, left coronary sinus; MDCT, multidetector computed tomography; NCS, non-coronary sinus; RCS, right coronary sinus; SVA, sinus of Valsalva aneurysm; TTE, transthoracic echocardiography; TEE, transesophageal echocardiography.

imaging studies included: (I) TEE: 93.4\% (surgical group) vs. $22.2 \%$ (conservative group), $\mathrm{P}<0.001$; (II) MDCT: $61.8 \%$ (surgical group) vs. $37.0 \%$ (conservative group), $\mathrm{P}=0.041$; (III) CMR: $22.4 \%$ (surgical group) vs. $14.8 \%$ (conservative group), $\mathrm{P}=0.579$ (Table 2 ). The vast majority of TEE studies (85\%) were performed as intraoperative studies. Because three-dimensional TEE was not routinely performed prior to 2008 , it was utilized in $37 \%$ of overall cases. Similarly, we have observed a higher use of tomographic imaging, particularly MDCT, in the more recent years included in the current study. MDCT was used in $55 \%$ of the study cohort, and out of 57 subjects undergoing MDCT, only 17 (29.8\%) patients underwent MDCT before 2007. Utilization of CMR in the current study may also have affected the utilization of MDCT, since CMR was utilized in $21.7 \%$ of the subjects that did not undergo MDCT. Thirteen-point-nine percent of the patients who did not undergo either MDCT or CMR imaging had serum creatinine levels $\geq 2.00 \mathrm{mg} / \mathrm{dL}$.

The median number of imaging modalities utilized was significantly higher in the surgical group, compared to the conservative group $\{3[4]$ vs. 2 [2], $\mathrm{P}<0.001\}$. TTE was used as the sole imaging modality in $10.7 \%$ cases $(37.0 \%$ in the conservative group $v s .1 .3 \%$ in the surgical group, $\mathrm{P}<0.001)$. 


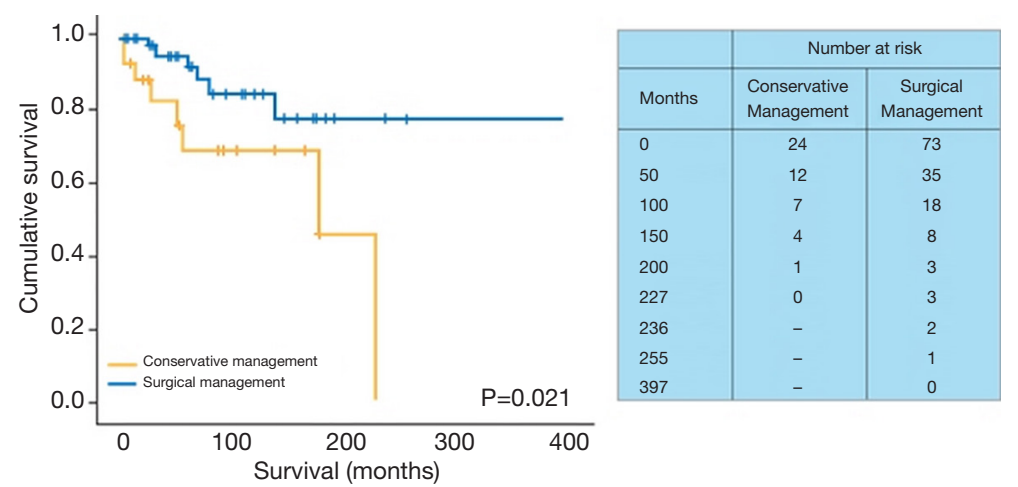

Figure 4 Kaplan-Meier survival analysis of SVA patients, stratified by management strategy: Blue curve-patients who underwent surgical management ( $n=73,7$ events); yellow curve-patients who underwent conservative management ( $n=26,8$ events). Note that follow-up data for the purpose of Kaplan-Meier analysis were based on 99 cases, since four patients were lost to follow-up (no subsequent clinic visits, and the mortality status was unknown). SVA, sinus of Valsalva aneurysm.

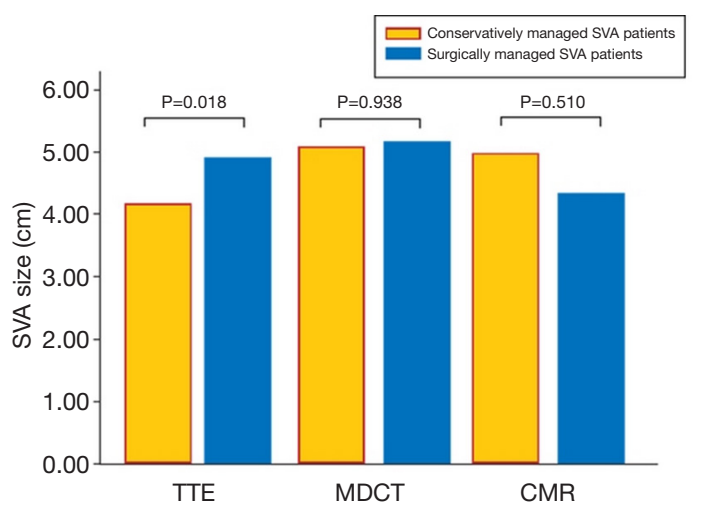

Figure 5 Comparison of SVA measurements using multimodality cardiovascular imaging in the conservative $v s$. surgical groups. A comparison for TEE is not shown, because there were only two patients undergoing TEE in the conservative management group. CMR, cardiac magnetic resonance; MDCT, multidetector computed tomography; SVA, sinus of Valsalva aneurysm; TTE, transthoracic echocardiography.

Complementary imaging modalities were TEE, MDCT and CMR. The combination of TTE, TEE and MDCT was more commonly performed in the surgical group (40.8\% vs. $11.1 \%$ in the conservative group, $\mathrm{P}=0.004)$. Other combinations of imaging modalities included: (I) TTE and TEE $(3.7 \%$ in the conservative group $v s .14 .5 \%$ in the surgical group, $\mathrm{P}=0.177)$; (II) TTE, TEE and CMR (3.7\% in the conservative group vs. $9.2 \%$ in the surgical group, $\mathrm{P}=0.677)$; (III) TTE and MDCT (14.8\% in the conservative group vs. $5.3 \%$ in the surgical group, $\mathrm{P}=0.202$ ); (IV) TTE and CMR $(3.7 \%$ in the conservative group vs. $0 \%$ in the surgical group, $\mathrm{P}=0.262)$.

At diagnosis, the maximum sinus-to-sinus SVA diameters were: $4.90(3.10) \mathrm{cm}$ (surgical group) vs. $4.20(3.20) \mathrm{cm}$ (conservative group) by TTE, $\mathrm{P}=0.018 ; 5.50(4.00) \mathrm{cm}$ (surgical group) vs. $5.30(0.20) \mathrm{cm}$ (conservative group) by TEE, $\mathrm{P}=0.725 ; 5.20(3.70) \mathrm{cm}$ (surgical group) vs. $5.10(3.90) \mathrm{cm}$ (conservative group) by MDCT, $\mathrm{P}=0.938$; and $4.40(2.80) \mathrm{cm}$ (surgical group) vs. $5.00(3.70) \mathrm{cm}$ (conservative group) by CMR, $\mathrm{P}=0.510$ (Table 2) (Figure 5).

Compared to patients with non-ruptured SVAs, patients presenting with ruptured SVAs had larger SVA diameters on TTE [5.20 (2.80) cm vs. $4.80(3.30) \mathrm{cm}$ for non-ruptured SVAs, $\mathrm{P}=0.039]$. SVA diameters measured by TEE, MDCT and CMR were also larger for ruptured SVAs, although the differences did not reach statistical significance [TEE: 5.60 (2.80) cm vs. 5.30 (4.00) cm, P=0.435; MDCT: $5.90(3.70) \mathrm{cm}$ vs. 5.15 (3.80) cm for non-ruptured SVAs, $\mathrm{P}=0.385$; CMR: 5.00 (3.70) $\mathrm{cm}$ vs. 4.80 (2.80) cm for non-ruptured SVAs, $\mathrm{P}=0.661]$.

A moderate correlation between TTE and TEE measurements were noted [Spearman's rho $(\rho)=0.609$, $\mathrm{P}<0.001]$. TEE measurements also showed moderate correlations with MDCT $(\rho=0.530, P=0.001)$ and $C M R$ $(\rho=0.667, P=0.050)$ measurements. MDCT measurements showed moderate correlations with CMR measurements $(\rho=0.646, P=0.043)$ (Table 3). Echocardiographic characteristics of the study cohort are shown in Table S3.

\section{Independent associations of SVA rupture}

Using a multivariable binomial logistic regression 
Table 3 Correlations between SVA sizes measured by TTE, TEE, MDCT and CMR imaging

\begin{tabular}{|c|c|c|c|c|}
\hline Imaging modalities & TTE & TEE & MDCT & CMR \\
\hline Spearman correlation coefficient $(\rho)$ & 1.000 & 0.609 & 0.574 & 0.412 \\
\hline$P$ value & - & $<0.001^{*}$ & $<0.001^{*}$ & 0.162 \\
\hline \multicolumn{5}{|l|}{ TEE } \\
\hline$P$ value & $<0.001^{*}$ & - & $0.001^{*}$ & $0.050^{*}$ \\
\hline \multicolumn{5}{|l|}{ MDCT } \\
\hline Spearman correlation coefficient $(\rho)$ & 0.574 & 0.530 & 1.000 & 0.646 \\
\hline$P$ value & $<0.001^{*}$ & $0.001^{*}$ & - & $0.043^{*}$ \\
\hline $\mathrm{P}$ value & 0.162 & $0.050^{*}$ & $0.043^{*}$ & - \\
\hline
\end{tabular}

*, $\mathrm{P}$ value $<0.05$ denotes statistical significance. CMR, cardiac magnetic resonance imaging; MDCT, multidetector computed tomography; SVA, sinus of Valsalva aneurysm; TTE, transthoracic echocardiography; TEE, transesophageal echocardiography.

model incorporating LVEF, aortic root dimension on echocardiography, a history of statin use, angiotensinconverting enzyme inhibitor use and beta-blocker use (variables that had a $\mathrm{P}$ value $<0.20$ in the univariable binomial logistic regression analysis results), LVEF (OR: $1.240,95 \%$ CI: $1.059-1.452, \mathrm{P}=0.008)$ and a history of statin use (OR: $0.175,95 \%$ CI: $0.042-0.729, \mathrm{P}=0.017$ ) were found to have an independent association with SVA rupture at presentation.

\section{Predictors of survival at follow-up}

Using Cox regression analysis, a multivariable model incorporating age, male gender, a history of congestive heart failure (CHF), a history of diabetes mellitus, a history of stroke, a history of statin use, a history of chronic obstructive pulmonary disease, surgical management (as opposed to conservative management), creatinine levels and right ventricular systolic pressure (variables that had a $\mathrm{P}$ value $<0.20$ in the univariable Cox regression analysis results), a history of CHF (HR: 4.953, 95\% CI: $1.131-$ 21.681, $\mathrm{P}=0.034)$ and higher right ventricular systolic pressure (HR: 1.048, 95\% CI: 1.020-1.077, $\mathrm{P}=0.001$ ) were found to be statistically significant independent predictors of increased mortality at follow-up.

\section{Discussion}

Our study reports the outcomes of imaging-guided SVA management over a 20 -year period at a high-volume center. Unique to this study is the inclusion of patients with SVAs who were conservatively managed. To the best of our knowledge, this is the largest cohort study reporting the utility of multimodality imaging in managing SVAs.

In our study, SVA patients were relatively older (median age: 58 years) compared to prior studies $(4,5)$. The higher median age of our cohort may partly reflect the changing demographics of the ageing population. Our observation that there were more men presenting with SVAs $(68.9 \%$ of our cohort) was consistent with prior reports $(4,5)$. Although SVAs are reported to have a higher prevalence in Asian and Far Eastern populations (6), the majority of our patients were Caucasian.

Additional congenital heart lesions were found in 50.5\% of the cohort. The most common lesion was BAV (26.2\%) similar to Takach et al. (4) reporting BAV as the most common associated congenital heart disease despite with a lower prevalence (16.3\%). The overall prevalence of VSD was lower in our surgical group $(21.1 \%)$ compared with previous reports of surgical cohorts of ruptured SVAs that ranged from $36.4 \%$ to $46.3 \%(5,7,8)$. The relatively lower prevalence of VSD in our study cohort may potentially be 
explained by differences in ethnicity (6). Right coronary sinus was most frequently affected by SVA (56.3\%), followed by the non-coronary sinus (35.9\%) and left coronary sinus $(7.8 \%)$, in agreement with other published SVA cohorts $(4,5,7,8)$.

A key strength of our study is highlighting the utility of echocardiography and multimodality imaging in the diagnosis and management of SVAs. We speculate that during the study period reported by Takach et al. (4) (19561997), most of the advanced multimodality cardiovascular imaging modalities were not routinely available. Therefore, these imaging techniques did not become incorporated as part of the diagnostic and surveillance strategy for patients with SVAs. In the contemporary era, we have found that non-invasive multimodality cardiovascular imaging, led by echocardiography, plays key roles in diagnosing SVAs (1). TTE was invariably used as the first-line imaging modality in the diagnosis of SVAs (100\% in the surgical group, $92.6 \%$ in the conservative group), due to its availability, portability and safety. TEE was significantly more frequently utilized in the surgical group $(\mathrm{P}<0.001)$, for intra-operative guidance, and to assess for SVA related complications. Tomographic imaging with MDCT and CMR, was utilized to provide complementary information. MDCT was used in $55.3 \%$ of the cohort, with a significantly higher proportion of patients in the surgical group undergoing MDCT (61.8\% vs. $37.0 \%$, $\mathrm{P}=0.041)$. CMR and invasive aortography of the SVAs were used in $20.4 \%$ and $19.4 \%$ of cases, respectively. The increased use of MDCT, compared to CMR, particularly in the surgical group, may be partly explained by the superior ability of MDCT to assess non-cardiac anatomy, as well as the speed of image acquisition for MDCT.

SVA dimensions were overall smaller on TTE, compared to tomographic imaging (Table 2, Figure 5). First, a smaller value may be obtained on TTE due to differences in measurement techniques. Leading edge-to-leading edge (L-L) convention is recommended for measurement of the aortic root using echocardiography (9). In comparison, The Society of Thoracic Surgeons Clinical Practice Guidelines published in 2013, stated that the external diameters of the aorta are measured by MDCT and CMR, potentially explaining a smaller aortic dimension obtained by echocardiography (10). It should be noted that nearly all patients underwent TTE, and only $55.3 \%$ and $20.4 \%$ of patients underwent MDCT and CMR, respectively. Therefore, patients who underwent tomographic imaging assessment may have been "selected" out to have larger SVA diameters.
Limited evidence exists comparing aortic root measurements between TTE and MDCT/CMR in patients with aortopathy. Tamborini et al. (11) reported the mean aortic root at the level of sinuses of Valsalva was $43.4 \mathrm{~mm}$ by TTE $v s .44 .8 \mathrm{~mm}$ on MDCT in 44 patients with known ascending aortic dilatation. Nejatian et al. (12) compared TTE and CMR aortic root measurements in 41 patients with either a connective tissue disorder $(n=22)$ or BAV $(n=19)$, which showed significantly smaller diameters obtained on TTE by 2.0-2.7 mm ( $\mathrm{P}<0.0001)$.

We documented different patterns of surgical techniques in the contemporary era compared to previous data by Takach et al. (4) and Luo et al. (5). We demonstrated lower in-hospital mortality for these procedures $(1.3 \%)$, with the only in-hospital death being related to endocarditis. Takach et al. (4) reported an in-hospital mortality of $3.9 \%$, and Sarikaya et al. (8) reported in-hospital mortality of $3.6 \%$. Our study is the first to demonstrate the mediumterm outcomes in conservatively managed SVA patients. Although the survival rate was significantly lower in the conservative group, none of the deaths in this group was related directly to SVAs. This may be due to the older patient profile in the conservative group. The estimated survival rate at 31 months was excellent at $94.0 \%$ in the surgical group.

Our study is the first to assess the independent association between baseline demographic, clinical and imaging parameters with SVA rupture at presentation. Importantly, LVEF was the only baseline imaging parameter that was independently associated with an increased probability of SVA rupture at presentation (OR: 1.240, 95\% CI: 1.059-1.452, $\mathrm{P}=0.008)$. A history of statin use was significantly associated with a reduced likelihood of SVA rupture at presentation. The impact of statin treatment on ascending aorta aneurysms was evaluated in a propensity score-matched case-control study (13), which showed reduced ascending aorta aneurysm growth rate in the statin treatment group $(\mathrm{n}=329)$ compared to no statin use $(\mathrm{n}=329)$ at 3 -year follow-up $(\mathrm{P}<0.001)$. Three-year survival outcomes for the composite outcome (death, dissection/ rupture, need for operative repair) was also significantly better in the statin group (HR: 0.69, 95\% CI: 0.47-1.01). However, there are currently no data on the efficacy of statin treatment in SVAs.

\section{Limitations}

The analysis is limited by the retrospective nature of the 
study. Follow-up data for patients who had long-term surveillance at other centers may have been missed. Being a single center with high surgical volumes and expertise, this study is also subject to selection bias. However, due to the rare nature of the condition, this study is the largest contemporary cohort study outlining the contemporary management of SVAs, guided by echocardiography and multimodality imaging. This study could be further strengthened by prospective, multi-center data.

\section{Conclusions}

In a contemporary 20-year cohort, the outcomes for surgically managed patients with SVAs were excellent. For conservatively managed patients, no deaths or late complications were attributed to SVAs, although a higher mortality rate was observed. Multimodality cardiovascular imaging, led by echocardiography and complemented by MDCT and CMR, have helped guide the diagnosis and management of SVAs in the contemporary era.

\section{Acknowledgments}

Funding: None.

\section{Footnote}

Provenance and Peer Review: This article was commissioned by the editorial office, Cardiovascular Diagnosis and Therapy for the series "Heart Valve Disease". The article has undergone external peer review.

Reporting Checklist: The authors have completed the STROBE reporting checklist. Available at http://dx.doi. org/10.21037/cdt-20-630

Data Sharing Statement: Available at http://dx.doi. org/10.21037/cdt-20-630

Conflicts of Interest: All authors have completed the ICMJE uniform disclosure form (available at http://dx.doi. org/10.21037/cdt-20-630). The series "Heart Valve Disease" was commissioned by the editorial office without any funding or sponsorship. PS serves as the Editor- InChief of Cardiovascular Diagnosis and Therapy. BX served as the unpaid Guest Editor of the series. DK reports that she received a personal non-commercial grant from the Turkish Society of Cardiology, outside the submitted work.
SDF reports personal fees from Renova Therapeutics, Inc., Precision Image Analysis, Inc. and serves in a fiduciary capacity, such as an elected officer, director, or chief medical officer, for Cryothermic Systems, Inc. and Precision Image Analysis, Inc., outside the submitted work. LGS reports to receive or have the right to receive royalty payments for inventions or discoveries commercialized through Posthorax GmbH, outside the submitted work. The authors have no other conflicts of interest to declare.

Ethical Statement: The authors are accountable for all aspects of the work in ensuring that questions related to the accuracy or integrity of any part of the work are appropriately investigated and resolved. The study was approved by the institutional review board of the Cleveland Clinic (IRB number: 18-1445), and was compliant with the HIPAA (Health Insurance Portability and Accountability Act) regulations. The study was conducted in accordance with the Declaration of Helsinki (as revised in 2013). Individual informed consent was waived.

Open Access Statement: This is an Open Access article distributed in accordance with the Creative Commons Attribution-NonCommercial-NoDerivs 4.0 International License (CC BY-NC-ND 4.0), which permits the noncommercial replication and distribution of the article with the strict proviso that no changes or edits are made and the original work is properly cited (including links to both the formal publication through the relevant DOI and the license). See: https://creativecommons.org/licenses/by-nc-nd/4.0/.

\section{References}

1. Xu B, Kocyigit D, Betancor J, et al. Sinus of Valsalva Aneurysms: A State-of-the-Art Imaging Review. J Am Soc Echocardiogr 2020;33:295-312.

2. Chu SH, Hung CR, How SS, et al. Ruptured aneurysms of the sinus of Valsalva in Oriental patients. J Thorac Cardiovasc Surg 1990;99:288-98.

3. Mayer ED, Ruffmann K, Saggau W, et al. Ruptured aneurysms of the sinus of Valsalva. Ann Thorac Surg 1986;42:81-5.

4. Takach TJ, Reul GJ, Duncan JM, et al. Sinus of Valsalva aneurysm or fistula: management and outcome. Ann Thorac Surg 1999;68:1573-7.

5. Luo X, Zhang D, Li B, et al. Surgical repair of a ruptured congenital sinus of Valsalva aneurysm: 10-year experience with 286 cases. Eur J Cardiothorac Surg 2019;55:1211-8. 
6. Wang ZJ, Zou CW, Li DC, et al. Surgical repair of sinus of Valsalva aneurysm in Asian patients. Ann Thorac Surg 2007;84:156-60.

7. Xiao JW, Wang QG, Zhang DZ, et al. Clinical outcomes of percutaneous or surgical closure of ruptured sinus of Valsalva aneurysm. Congenit Heart Dis 2018;13:305-10.

8. Sarikaya S, Adademir T, Elibol A, et al. Surgery for ruptured sinus of Valsalva aneurysm: 25-year experience with 55 patients. Eur J Cardiothorac Surg 2013;43:591-6.

9. Lang RM, Badano LP, Mor-Avi V, et al. Recommendations for cardiac chamber quantification by echocardiography in adults: an update from the American Society of Echocardiography and the European Association of Cardiovascular Imaging. J Am Soc Echocardiogr 2015;28:1-39.e14.

Cite this article as: $\mathrm{Xu} \mathrm{B}$, Kocyigit D, Godoy-Rivas C, Betancor J, Rodriguez LL, Menon V, Jaber W, Grimm R, Flamm SD, Schoenhagen P, Svensson LG, Griffin BP. Outcomes of contemporary imaging-guided management of sinus of Valsalva aneurysms. Cardiovasc Diagn Ther 2021;11(3):770-780. doi: 10.21037/cdt-20-630
10. Svensson LG, Adams DH, Bonow RO, et al. Aortic valve and ascending aorta guidelines for management and quality measures. Ann Thorac Surg 2013;95:S1-66.

11. Tamborini G, Galli CA, Maltagliati A, et al. Comparison of feasibility and accuracy of transthoracic echocardiography versus computed tomography in patients with known ascending aortic aneurysm. Am J Cardiol 2006;98:966-9.

12. Nejatian A, Yu J, Geva T, et al. Aortic Measurements in Patients with Aortopathy are Larger and More Reproducible by Cardiac Magnetic Resonance Compared with Echocardiography. Pediatr Cardiol 2015;36:1761-73.

13. Angeloni E, Vitaterna A, Pirelli M, et al. Effects of statin therapy on ascending aorta aneurysms growth: A propensity-matched analysis. Int J Cardiol 2015;191:52-5. 
Supplementary

Table S1 Types of congenital heart disease associated with sinus of Valsalva aneurysms in the study cohort

\begin{tabular}{|c|c|c|c|c|}
\hline Type of congenital heart disease, n (\%) & Entire cohort $(n=103)$ & Conservative group $(n=27)$ & Surgical group $(n=76)$ & $P$ value \\
\hline Isolated VSD & $9(8.7)$ & 0 & $9(11.8)$ & \\
\hline Isolated ASD & $1(1.0)$ & 0 & $1(1.3)$ & \\
\hline VSD and PFO & $3(2.9)$ & 0 & $3(3.9)$ & \\
\hline VSD, BAV and PFO & $1(1.0)$ & 0 & $1(1.3)$ & \\
\hline VSD and MVP & $1(1.0)$ & 0 & $1(1.3)$ & \\
\hline VSD and Gerbode defect & $1(1.0)$ & 0 & $1(1.3)$ & \\
\hline PFO and MVP & $1(1.0)$ & 0 & $1(1.3)$ & \\
\hline Isolated MVP & $1(1.0)$ & 0 & $1(1.3)$ & \\
\hline Isolated PFO & $7(6.8)$ & $3(11.1)$ & $4(5.3)$ & \\
\hline Coarctation of the aorta and BAV & $1(1.0)$ & $1(3.7)$ & 0 & \\
\hline ASD, BAV and PFO & $1(1.0)$ & 0 & $1(1.3)$ & \\
\hline ASD, BAV & $1(1.0)$ & 0 & $1(1.3)$ & \\
\hline
\end{tabular}

ASD, atrial septal defect; BAV, bicuspid aortic valve; MVP, mitral valve prolapse; PFO, patent foramen ovale; VSD, ventricular septal defect. 
Table S2 Early and late complications in the surgical group (n=76)

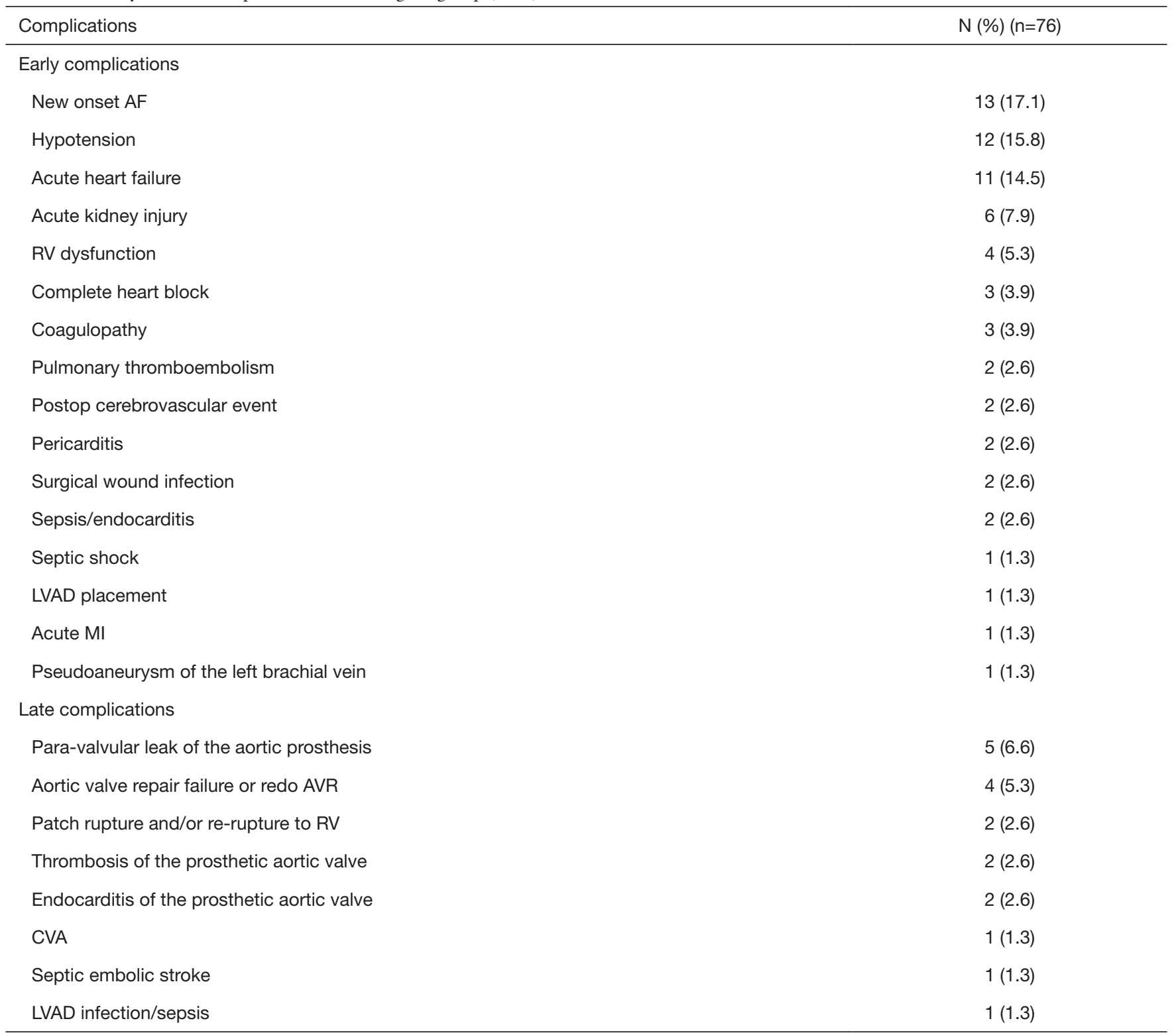

AF, atrial fibrillation; AVR, aortic valve replacement; CVA, cerebrovascular accident; LVAD, left ventricular assist device; MI, myocardial infarction; RV, right ventricle. 
Table S3 Echocardiographic characteristics of the study cohort

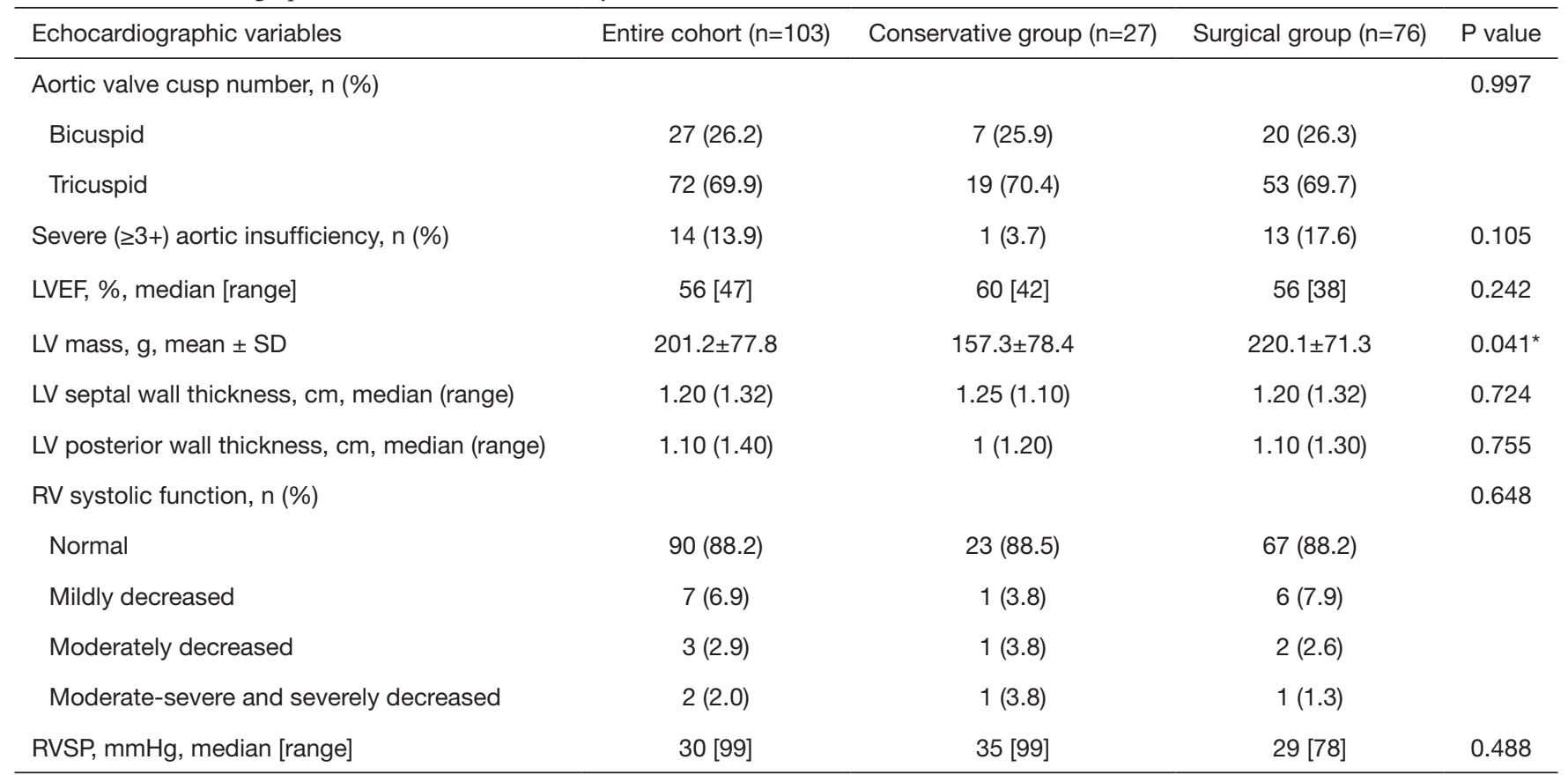

*, $\mathrm{P}$ value $<0.05$ denotes statistical significance. LV, left ventricular; LVEF, left ventricular ejection fraction; RV, right ventricular; RVSP, right ventricular systolic pressure; SD, standard deviation. 